\title{
Ultrasonography Evaluation of Vulnerable Vessels Around Cervical Nerve Roots During Selective Cervical Nerve Root Block
}

\author{
Hoon Hoon Lee, $\mathrm{MD}^{1}$, Donghwi Park, $\mathrm{MD}^{2}$, Yoongul Oh, $\mathrm{MD}^{3}$, Ju Seok Ryu, MD, PhD ${ }^{1}$ \\ ${ }^{1}$ Department of Rehabilitation Medicine, Seoul National University Bundang Hospital, Seoul National University \\ College of Medicine, Seongnam; ${ }^{2}$ Department of Rehabilitation Medicine, Daegu Fatima Hospital, Daegu; \\ ${ }^{3}$ Department of Rehabilitation Medicine, CHA Bundang Medical Center, CHA University, Seongnam, Korea
}

\begin{abstract}
Objective To evaluate the prevalence of vulnerable blood vessels around cervical nerve roots before cervical nerve root block in the clinical setting.

Methods This retrospective study included 74 patients with cervical radiculopathy who received an ultrasonography-guided nerve block at an outpatient clinic from July 2012 to July 2014. Before actual injection of the steroid was performed, we evaluated the vulnerable blood vessels around each C5, C6, and C7 nerve root of each patient's painful side, with Doppler ultrasound.

Results Out of 74 cases, the C5 level had 2 blood vessels (2.7\%), the C6 level had 4 blood vessels (5.45\%), and the C7 level had 6 blood vessels $(8.11 \%)$ close to each targeted nerve root. Moreover, the C5 level had 2 blood vessels (2.7\%), the C6 level 5 blood vessels (6.75\%), and the C7 level had 4 blood vessels (5.45\%) at the site of an imaginary needle's projected pathway to the targeted nerve root, as revealed by axial transverse ultrasound imaging with color Doppler imaging. In total, the C5 level had 4 blood vessels (5.45\%), the C6 level 9 blood vessels (12.16\%), and the C7 level 10 had blood vessels (13.51\%) either at the targeted nerve root or at the site of the imaginary needle's projected pathway to the targeted nerve root. There was an unneglectable prevalence of vulnerable blood vessels either at the targeted nerve root or at the site of the needle' projected pathway to the nerve root. Also, it shows a higher prevalence of vulnerable blood vessels either at the targeted nerve root or at the site of an imaginary needle's projected pathway to the nerve root as the spinal nerve root level gets lower.

Conclusion To prevent unexpected critical complications involving vulnerable blood vessel injury during cervical nerve root block, it is recommended to routinely evaluate for the presence of vulnerable blood vessels around each cervical nerve root using Doppler ultrasound imaging before the cervical nerve root block, especially for the lower cervical nerve root level.
\end{abstract}

Keywords Ultrasonography, Intervertebral disc displacement, Blood vessels, Epidural injections

Received March 14, 2016; Accepted July 19, 2016

Corresponding author: Ju Seok Ryu

Department of Rehabilitation Medicine, Seoul National University Bundang Hospital, Seoul National University College of Medicine, 82 Gumi-ro 173beon-gil, Bundang-gu, Seongnam 13620, Korea. Tel: +82-31-787-7739, Fax: +82-31-787-4051, E-mail: jseok337@snu.ac.kr

ORCID: Hoon Hoon Lee (http://orcid.org/0000-0002-5028-4318); Donghwi Park (http://orcid.org/0000-0002-7724-4682); Yoongul Oh (http://orcid. org/0000-0003-4324-9765); Ju Seok Ryu (http://orcid.org/0000-0003-3299-3038).

@ This is an open-access article distributed under the terms of the Creative Commons Attribution Non-Commercial License (http://creativecommons.org/ licenses/by-nc/4.0) which permits unrestricted noncommercial use, distribution, and reproduction in any medium, provided the original work is properly cited. Copyright $\odot 2017$ by Korean Academy of Rehabilitation Medicine 


\section{INTRODUCTION}

Fluoroscopy-guided cervical nerve root block is conventionally used for managing cervical radiculopathy. In general, the needle approach in the posterior aspect of the foramen has been popularly used to avoid the penetration of critical blood vessels such as the vertebral artery [1]. However, always underlying such a procedure is the risk of critical complication of unintentional blood vessel injury despite following strict guidelines, such as carefully performing a fluoroscopy-guided needle approach in the posterior aspect of the foramen to minimize the inherent risks.

A survey by Ma et al. [2] of 1,036 consecutive nerve blocks (specifically, fluoroscopically-guided extraforaminal cervical nerve blocks) revealed a complication rate of $1.64 \%$. Most of the serious reported complications were commonly associated with intravascular injections, which resulted in infarction of the spinal cord and the brainstem [3].

In previous studies, the authors suggested that unexpected anatomical variations of cervical arteries might be the cause of unintended intravascular injections [3]. In a cadaveric study by Huntoon [4], 7 out of 95 cases $(7.3 \%)$ showed that some critical artery branches extending from the vertebral, ascending, and deep cervical arteries were located in the posterior aspects of the foramen, which are vulnerable to the conduction of routine transforaminal epidural steroid injections (Fig. 1). Also, the Hoeft et al. [5] cadaveric study showed an anatomical variation of the radicular artery entering the cervical intervertebral foramen. In the case series study showing the feasibility of administering an ultrasound-guided cervical nerve root block for 10 patients, Narouze et al. [6] reported that 2 patients had critical arteries at the posterior aspects and 1 patient had a critical one continuing medially into the foramen.

In this study, the authors suggest that the anatomical variation of cervical arteries is much more than has previously been known [6]. Until now, there has been no definitive report regarding the prevalence of critical blood vessels around each cervical nerve root level in the clinical setting.

So in this study, we have tried to evaluate the prevalence of vulnerable blood vessels around each cervical nerve root prior to cervical nerve block in the clinical setting.

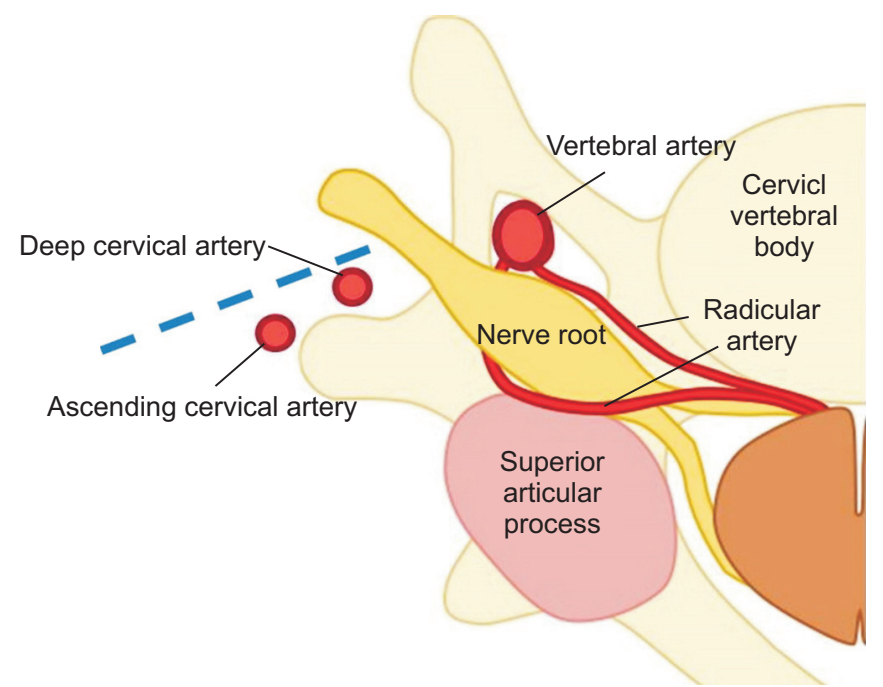

Fig. 1. Schematic illustration of the possible regional vascular anatomy around the cervical nerve root. There are reports that some critical artery branches (extending from the ascending and deep cervical arteries and the radicular artery) are located in the posterior aspects of the foramen-which can be injured when conducting routine transforaminal epidural steroid injections. The dotted line indicates an imaginary needle's projected pathway to the target of injection during a conventional needle approach.

\section{MATERIALS AND METHODS}

\section{Participants}

This study included 74 patients with cervical radiculopathy who received ultrasonography-guided nerve block at an outpatient clinic from July 2012 to July 2014 in CHA Bundang Medical Center and Seoul National University Bundang Hospital. Medical records and ultrasound images were retrospectively analyzed.

\section{Ultrasound evaluation}

Before the actual injection of the steroid was performed, we evaluated the vulnerable blood vessels around each C5, C6, and C7 nerve root of the patient's painful side, with Doppler ultrasound imaging. Ultrasound evaluations were performed using a standard ultrasound device (Philips iU22 DS; Philips Medical Systems, Cleveland, $\mathrm{OH}, \mathrm{USA}$ ) and a high-frequency linear transducer (5-12 $\mathrm{MHz}$ ). With patients being placed in a supine position, their heads were rotated $30^{\circ}$ to $40^{\circ}$ away from the painful side [7]. 
To determine spinal level, two methods were used. First, to determine the $\mathrm{C} 7$ level, the transducer scanned the lateral aspect of the neck transversally to identify the transverse process. The $\mathrm{C} 7$ transverse process differs from that of others by its having no prominent anterior tubercle [8]. Another way is to follow the vertebral artery, which runs anteriorly at the $\mathrm{C} 7$ level till arriving at the C6 level, where it enters the foramen in $90 \%$ of cases [9].

\section{Definition of vulnerable blood vessel}

The term 'vulnerable blood vessel' was defined as being an artery within $2 \mathrm{~mm}$ distance from the target of injection or located at the site of the projected pathway of the imaginary needle if it were to be placed in the posterior aspects of the intervertebral foramen [4].

\section{Statistical analysis}

The results are presented in terms of numbers and percentages (\%). Statistical analysis was performed using SPSS ver. 21.0 (IBM, Armonk, NY, USA).

\section{RESULTS}

\section{Demographics}

Total of 74 patients were included in the study, with an average age of 54.64 years (range, 28-77 years). Vulnerable blood vessels around each C5, C6, and C7 nerve root of the patient's painful side were all evaluated with Doppler ultrasound imaging, resulting in imaging of 74 cases of C5, C6, and C7 each.

\section{Evaluation of vulnerable blood vessels}

In all cases, cervical transverse processes and spinal nerve levels were well identified in the short axial ultrasound view, with the anterior tubercle and the posterior tubercle well visualized as the '2-humped camel' sign (Fig. 2).

Short axial view of color Doppler ultrasound imaging revealed the presence of a vulnerable blood vessel at the targeted nerve root in the intervertebral foramen at each nerve level (Figs. 2, 3). Out of 74 cases, the C5 level had 2 blood vessels (2.7\%), the C6 level had 4 blood vessels (5.45\%), and the C7 level had 6 blood vessels ( $8.11 \%)$ close to each targeted nerve root (Table 1). The presence of vulnerable blood vessels at the site of an imaginary needle's projected pathway to the targeted nerve root in the intervertebral foramen were also revealed by short axial color Doppler ultrasound imaging (Fig. 4). Out of 74 cases each, the $\mathrm{C} 5$ level had 2 blood vessels $(2.7 \%)$, the C6 level had 5 blood vessels (6.75\%), and the C7 level had 4 blood vessels (5.45\%) at the site of the imaginary needle's projected pathway to the targeted nerve root (Table 1). These blood vessels could have easily been injured by a conventional fluoroscopy needle approach.

In total, the C5 level had 4 blood vessels (5.45\%), the C6 level had 9 blood vessels (12.16\%), and the C7 level had 10 blood vessels (13.51\%) either at the targeted nerve root or at the site of the imaginary needle's projected pathway to the targeted nerve root (Table 1).
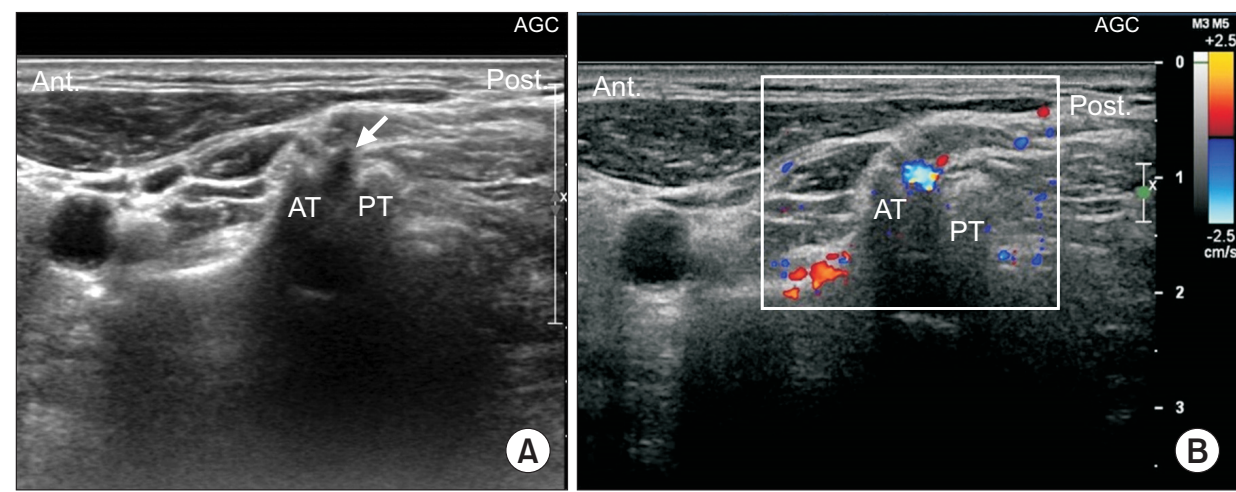

Fig. 2. (A) Short axial ultrasound image showing the C5 transverse process. Note the anterior tubercle (AT) and the posterior tubercle (PT) as the '2-humped camel' sign. Solid arrows indicate nerve root. (B) Short axial view of ultrasound imaging (with color Doppler) revealing a blood vessel located at the site of the targeted nerve root in the intervertebral foramen. 

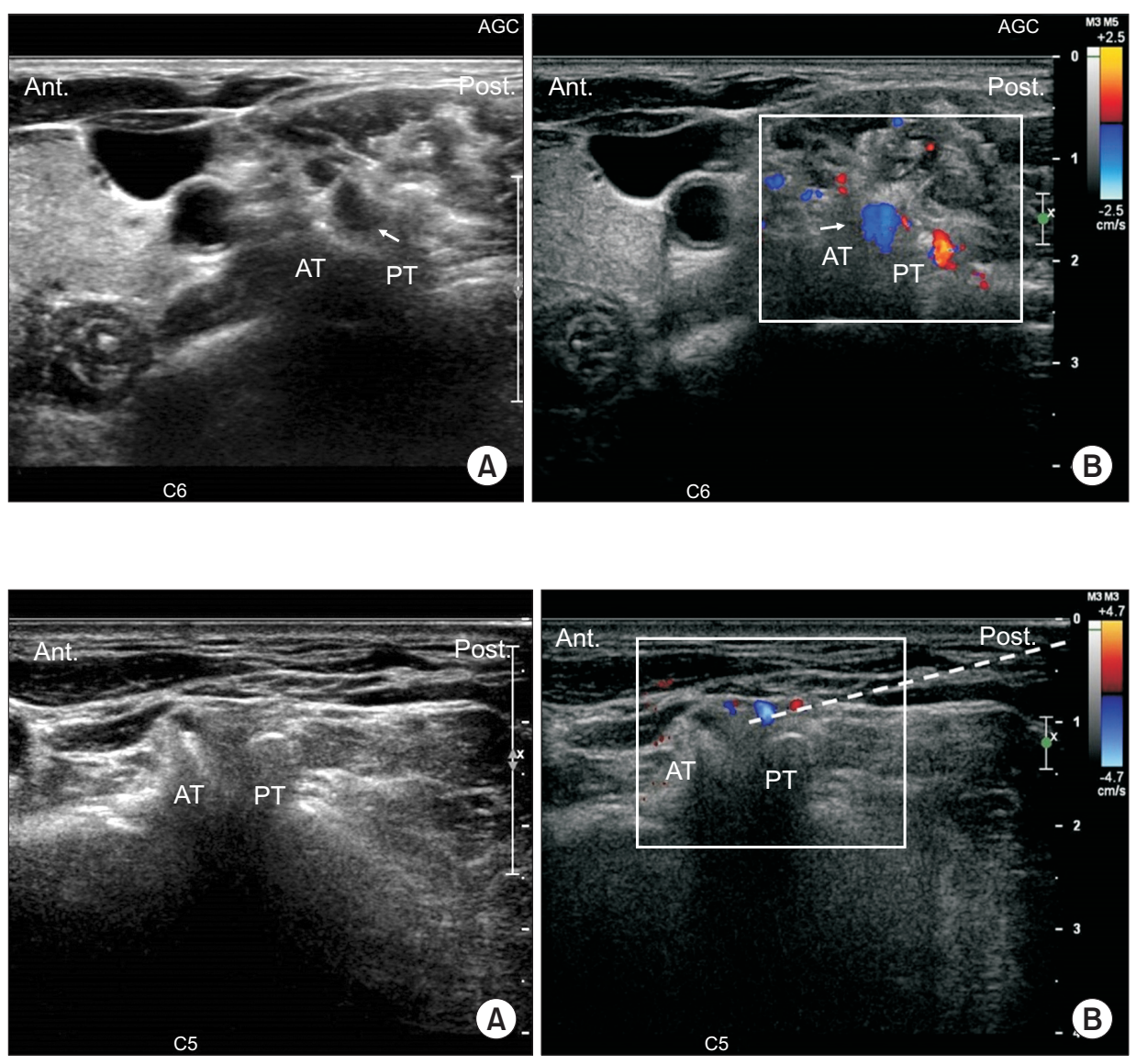

Fig. 3. (A) Short axial ultrasound image showing the C6 transverse process. Solid arrow indicates nerve root. (B) Short axial transverse ultrasound imaging (with color Doppler) revealing a blood vessel at the targeted nerve root (indicated by a solid arrow) in the intervertebral foramen. AT, anterior tubercle; PT, posterior tubercle.

Fig. 4. (A) Another short axial view of ultrasound image showing the C5 transverse process. (B) Axial transverse ultrasound imaging (with color Doppler imaging) revealing a blood vessel at the imaginary needle's projected pathway (dotted line) to the targeted nerve root in the intervertebral foramen. AT, anterior tubercle; PT, posterior tubercle.
Table 1. Distribution of vulnerable blood vessels around each spinal nerve root

\begin{tabular}{llcl}
\hline $\begin{array}{c}\text { Spinal nerve } \\
\text { level }\end{array}$ & $\begin{array}{c}\text { Around } \\
\text { target }^{\text {a) }}\end{array}$ & $\begin{array}{c}\text { At needle } \\
\text { pathway }^{\text {b) }}\end{array}$ & Total \\
\hline C5 & $2(2.7)$ & $2(2.7)$ & $4(5.45)$ \\
C6 & $4(5.45)$ & $5(6.75)$ & $9(12.16)$ \\
C7 & $6(8.11)$ & $4(5.45)$ & $10(13.51)$ \\
Total & 12 & 11 & 23 \\
Mean\% & 5.41 & 4.95 & 10.36 \\
\hline
\end{tabular}

Values are presented as number (\%).

${ }^{a)}$ Blood vessel within $2 \mathrm{~mm}$ distance from the target of

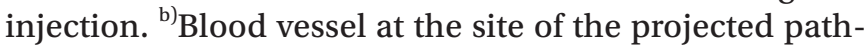
way of an imaginary needle in the posterior aspects of the intervertebral foramen.

\section{DISCUSSION}

Until now, there has been no study showing the prevalence of vulnerable blood vessels around each cervical nerve at the root level, by ultrasound, in the clinical setting. While the previous studies of researchers such as
Huntoon [4] and Hoeft et al. [5] have shown blood vessel distributions in cadavers, what was remarkable about this study is that it tried to actionably reveal the actual distribution and prevalence of such blood vessels in the clinical setting.

Cervical spinal arterial branches arise mainly from the vertebral, ascending cervical, superior intercostal, and deep cervical arteries. These spinal arterial branches pass through the intervertebral foramina just adjacent to the spinal nerves and continue as radicular arteries. Then, the radicular artery penetrates the dura to supply the nerve roots and to join the anterior and posterior spinal arteries. This radicular artery is a major source of blood for the nerve root and the spinal cord-so damage to this artery, such as through intra-articular injection, can lead to serious complications [5].

In the study by Baker et al. [10], even real-time contrast fluoroscopy was considered to be insufficient, so it is recommended to check arterial filling using real-time fluoroscopy with digital subtraction, for the detection of unintentional intravascular injections. Also, the fluoroscopy 
method can increase the patient's and the operator's exposure to radiation. So to overcome the shortcomings of fluoroscopy, ultrasonography has recently and rapidly been becoming an alternate technique for intervention [11]. In the previous study, showing the effectiveness of ultrasound as compared with fluoroscopy, ultrasoundguided intervention showed comparatively equal efficacy in pain relief and comparatively equal efficacy in functional assessment with fluoroscopy-guided injection $[6,12]$. Other than the advantage of involving no radiation exposure, ultrasonography has the capability of visualizing structures such as soft tissues, blood vessels, nerves, and needle-tip placement on real-time imaging scans; and it can also allow for the observation of the spread of injectate [6].

Back to the results of this study: There was an unneglectable prevalence of vulnerable blood vessels either at the targeted nerve root or at the site of the imaginary needle's projected pathway to the nerve root. This result means that if transforaminal epidural injection with fluoroscopy were to be done as the usual protocol (to approach from the posterior aspect) without ultrasound examination in those patients, then the likelihood of injuring a critical blood vessel is high. The likelihood that unexpectedly injuring a critical blood vessel located in the posterior aspect of a patient's foramen (either at the targeted nerve root or at the site of the imaginary needle's projected pathway to the nerve root) is approximately one out of 10. Also, it shows that lower the cervical spinal nerve roots, a higher prevalence of vulnerable blood vessels both at the target site and at the site of the imaginary needle's projected pathway to the nerve root. Therefore, needs to be more cautious while conducting lower cervical nerve root block and ultrasonography can be helpful to avoid the unintentional blood vessel injury.

Ultrasound, however, has some drawbacks such as limited visualization at deeper levels, especially under bony structures, and relative dependency on operator experience for fine imaging and intervention. In contrast to ultrasound, fluoroscopy-guided injection has the advantage of making bony structures clearly visible. By using ultrasound as a prior examination before fluoroscopyguided transforaminal injection, we can not only maximize the benefits of these two examinations, but we can also minimize the cost of catastrophic complications that patients could suffer.

Our present study had some limitations. Firstly, we only examined the C5-7 cervical root level, rather than the whole cervical level. However, the prevalence of C5-7 cervical radiculopathies accounts for about $80 \%$ of total cervical radiculopathies [13]. Considering the prevalence of cervical radiculopathies as shown in the previous study, our study result may be useful in clinical settings. Still, though, further evaluation of the upper cervical level may be needed. Secondly, the study lacks detailed clinical data, sociodemographic data, and long-term clinical outcome data. In this study, we could not evaluate the efficacy of ultrasound-guided cervical root block, but rather we only showed the prevalence of vulnerable arteries at each cervical root level. Therefore, assembling a conglomeration of more clinical data regarding longterm outcomes and correlating these outcomes with the prevalence of vulnerable arteries near the cervical root will enhance the value of the present study.

Also, this study did not distinguish between whether each proposed 'vulnerable blood vessel' was an artery or a vein. If it were an artery, we still could not recognize which artery it was and where it had come from. So in a future study, it would be more worthwhile to distinguish between arteries and veins and to trace each blood vessel to see where it branches from.

In conclusion, to prevent unexpected critical complications involving vulnerable blood vessel injury during the cervical nerve root block, it is recommended to routinely evaluate the vulnerable blood vessels around the cervical nerve root using Doppler ultrasound imaging prior to the fluoroscopy-guided cervical nerve root block-especially for lower cervical nerve root level injections.

\section{CONFLICT OF INTEREST}

No potential conflicts of interest relevant to this article.

\section{ACKNOWLEDGMENTS}

This research was supported by a Basic Science Research Program grant through the National Research Foundation of Korea (NRF) funded by the Ministry of Science, ICT and Future Planning (NRF-2013R1A1A1004622).

\section{REFERENCES}

1. Rathmell JP, Aprill C, Bogduk N. Cervical transforami- 
nal injection of steroids. Anesthesiology 2004;100: 1595-600.

2. Ma DJ, Gilula LA, Riew KD. Complications of fluoroscopically guided extraforaminal cervical nerve blocks: an analysis of 1036 injections. J Bone Joint Surg Am 2005;87:1025-30.

3. Tiso RL, Cutler T, Catania JA, Whalen K. Adverse central nervous system sequelae after selective transforaminal block: the role of corticosteroids. Spine J 2004;4:468-74.

4. Huntoon MA. Anatomy of the cervical intervertebral foramina: vulnerable arteries and ischemic neurologic injuries after transforaminal epidural injections. Pain 2005;117:104-11.

5. Hoeft MA, Rathmell JP, Monsey RD, Fonda BJ. Cervical transforaminal injection and the radicular artery: variation in anatomical location within the cervical intervertebral foramina. Reg Anesth Pain Med 2006;31:2704.

6. Narouze SN, Vydyanathan A, Kapural L, Sessler DI, Mekhail N. Ultrasound-guided cervical selective nerve root block: a fluoroscopy-controlled feasibility study. Reg Anesth Pain Med 2009;34:343-8.

7. Yamauchi M, Suzuki D, Niiya T, Honma H, Tachibana $\mathrm{N}$, Watanabe A, et al. Ultrasound-guided cervical nerve root block: spread of solution and clinical effect.
Pain Med 2011;12:1190-5.

8. Martinoli C, Bianchi S, Santacroce E, Pugliese F, Graif M, Derchi LE. Brachial plexus sonography: a technique for assessing the root level. AJR Am J Roentgenol 2002;179:699-702.

9. Matula C, Trattnig S, Tschabitscher M, Day JD, Koos WT. The course of the prevertebral segment of the vertebral artery: anatomy and clinical significance. Surg Neurol 1997;48:125-31.

10. Baker R, Dreyfuss P, Mercer S, Bogduk N. Cervical transforaminal injection of corticosteroids into a radicular artery: a possible mechanism for spinal cord injury. Pain 2003;103:211-5.

11. Hopkins PM. Ultrasound guidance as a gold standard in regional anaesthesia. Br J Anaesth 2007;98:299-301.

12. Jee H, Lee JH, Kim J, Park KD, Lee WY, Park Y. Ultrasound-guided selective nerve root block versus fluoroscopy-guided transforaminal block for the treatment of radicular pain in the lower cervical spine: a randomized, blinded, controlled study. Skeletal Radiol 2013;42:69-78.

13. Kondo K, Molgaard CA, Kurland LT, Onofrio BM. Protruded intervertebral cervical disk: incidence and affected cervical level in Rochester, Minnesota, 1950 through 1974. Minn Med 1981;64:751-3. 